\title{
Electrodeposition of Polypyrrole Nanowires within Vertically Oriented Mesoporous Silica Template
}

\author{
Hosuk Kang, Hochun Lee ${ }^{\dagger, *}$, and Juhyoun Kwak* \\ Molecular-Level Interface Research Center (MIRC), Department of Chemistry, Korea Advanced Institute of Science \\ and Technology (KAIST), Daejeon 305-701, Republic of Korea \\ ${ }^{\dagger}$ Department of Applied Chemistry, Kumoh National Institute of Technology, Gumi, Kyungbuk 730-701, Republic of Korea
}

(Received January 24, 2011 : Accepted February 8, 2011)

\begin{abstract}
Polypyrrole (Ppy) nanowire has been electrochemically synthesized via vertically oriented mesoporous silica template. The mesoporous template is also electrochemically deposited on indium tin oxide coated (ITO) glass from tetraethyl orthosilicate (TEOS) and cetyltrimethylammonium bromide (CTAB) surfactant. The highly ordered silica template is demonstrated to be $100 \sim 120 \mathrm{~nm}$ thick with the pores of $4 \sim 5 \mathrm{~nm}$ diameter by scanning electron microscope (SEM) and transmission electron microscope (TEM). Ppy is formed to fill pores of the silica template from pyrrole solution by electrochemical oxidation. The Ppy in Ppy/silica/ITO composite was found to exhibit reversible electrochemical activity, as characterized by cyclic voltammetry (CV).
\end{abstract}

Keywords : Polypyrrole, Nanowire, Mesoporous silica, Electrochemical capacitor

\section{Introduction}

Vertically oriented porous materials are promising templates for fabrication of nanowires and nanotubes. Various metal and conducting polymer (CP) nanowires can be synthesized by simple deposition into the porous template. Especially, extensive research on the fabrication of nanowires using porous anodic aluminum oxide (AAO) template has long been conducted. ${ }^{1,2}$ Recently, a novel method to prepare highly-ordered mesoporous silica thin film in which the direction of pores are normal to the substrate was reported by Walcarius group. ${ }^{3,4)}$ Novel synthesis and application is possible with the silica template due to its much smaller pores compared to AAO. Silica template with vertically aligned pores to the electrode surface has many advantages in terms of conductivity and modification of pores. Prussian blue $e^{5)}$ and polythiophene ${ }^{6}$ depositions into the silica template have been recently reported.

This study demonstrates Ppy nanowires embedded in the vertically oriented mesoporous silica template. The silica film is prepared by electrochemically-assisted method

*E-mail: dukelee@kumoh.ac.kr based on sol-gel process ${ }^{7,8)}$ from silica sol solution containing surfactant. In ethanol and water mixture solution, silica precursors and surfactants form mesostructures by self-assembly. ${ }^{9)}$ The mesostructures are mainly determined by the ratio of silica precursor and surfactant concentrations. Cylindrical mesostructure of silica-surfactant is formed by adjusting solution concentrations ${ }^{10)}$ and hydroxyl ions generated by cathodic potential catalyze sol-gel process. ${ }^{4)}$ Subsequently, Ppy is deposited into silica template by anodic oxidation from aqueous pyrrole solution.

\section{Experimental}

\subsection{Chemicals and preparation}

Tetraethylorthosilicate (TEOS, 99.0\%), cetyltrimethylammonium bromide (CTAB, 99.0\%), sodium nitrate (99.0\%), potassium ferricyanide $\left(\mathrm{K}_{3} \mathrm{Fe}(\mathrm{CN})_{6}, 99+\%\right)$, and pyrrole (99\%) were purchased from Aldrich(Seoul, Korea). Hydrochloric acid (min 35\%, Junsei), absolute ethanol (Merck) and ultrapure water ( $>18 \mathrm{M}$, Millipore system) were used. Indium tin oxide (ITO, $0.7 \mathrm{~mm}, 50 \Omega$ ) glass was purchased from JMI Korea.

The starting sol solutions were prepared from $0.1 \mathrm{M}$ $\mathrm{NaNO}_{3}$ aqueous solution. To $20 \mathrm{ml}$ of this solution, 
1.32 3 mmol of TEOS, $20 \mathrm{ml}$ ethanol, and CTAB were added to adjust the mole ratio of CTAB/TEOS to 0.32 . Before using, the sol solutions were stirred moderately and aged for 150 mins. Electrochemical experiments were conducted by the three electrode system using SCE as reference electrode, and platinum wire as counter electrode. Working electrode was immobilized and the surface area was limited by O-ring in Teflon cell. Cathodic potential was applied for growing of silica films and sol solution was immediately removed and washed by water after electrochemical treatment for inhibition of any further unexpected deposition of silica. After silica deposition, the electrodes were dried in $130^{\circ} \mathrm{C}$ for overnight for condensation and hardening the silica skeletons. The surfactants were removed by $0.1 \mathrm{M} \mathrm{HCl}$ ethanol solution for $5 \mathrm{~min}$ under mild stirring. Subsequently, washing by water and blowing by nitrogen steps were followed. Potentiostatic and galvanostatic method for deposition is tested in varying deposition time. $0.1 \mathrm{M}$ pyrrole, $0.2 \mathrm{M}$ $\mathrm{KCl}$ aqueous solution is used for Ppy deposition. Ppy is synthesized on silica-ITO composite electrode by galvanostatic oxidation.

\subsection{Characterization}

Cross-sectional SEM was employed to estimate the thickness of silica film and surface morphology is demonstrated by tilted SEM.

Silica film is mechanically scratched from ITO glass electrode and placed on TEM grid by water dispersion.

Ion accessibility through silica film is tested by cyclic voltammetric analysis using $0.1 \mathrm{mM}$ ferricyanide as redox probe. Electrolyte effect to ion accessibility is tested in various $0.01 \sim 0.1 \mathrm{M}$ of $\mathrm{KCl}$ supporting electrolyte. Reduction and oxidation of Ppy nanowires embedded in silica template is characterized by $\mathrm{CV}$ in $0.2 \mathrm{M} \mathrm{KCl}$ solution.

\section{Results and Discussion}

3.1. Characterization of mesoporous silica template Ordered mesoporous silica film was prepared on ITO glass electrode from aqueous TEOS solution containing CTAB by electrochemical method. The thickness of this silica film was determined as $100 \sim 120 \mathrm{~nm}$ from crosssectional SEM image (Fig. 1(a)). Aggregation-free silica surface was observed on ITO glass (Fig. 1(b)). TEM image supports the formation of well-ordered mesopores (dia. $4 \sim 5 \mathrm{~nm}$ ) in the silica template (Fig. 1(c)).
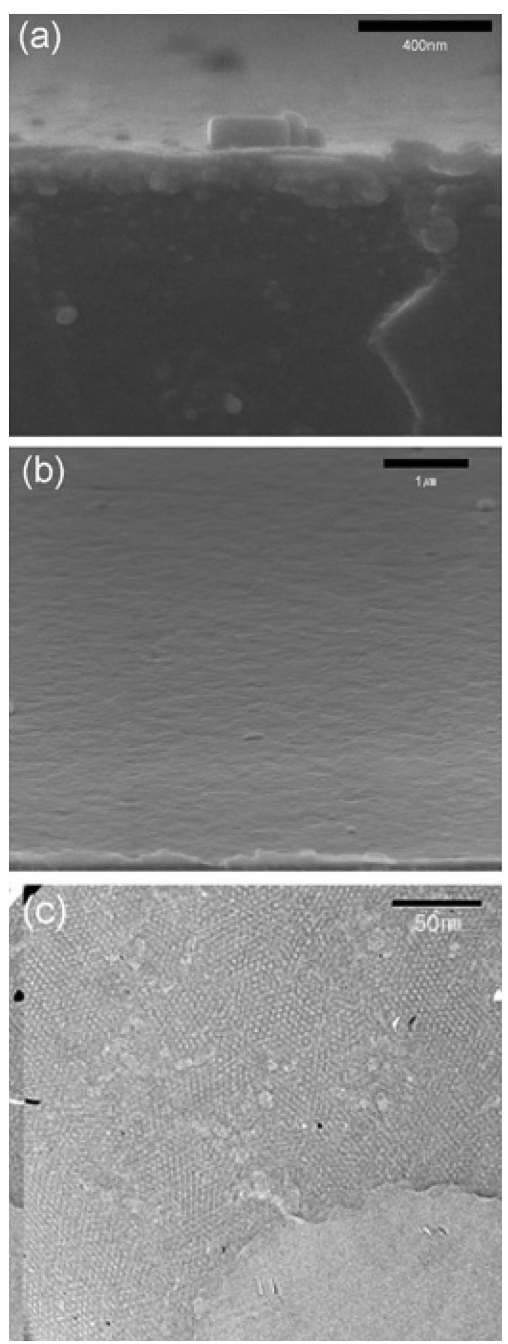

Fig. 1. (a) Cross-sectional image, (b) surface morphology obtained by tilted SEM and (c) TEM image of silica film.

Cyclic voltammetry (CV) of ferricyanide redox couple was performed to examine ion accessibility to the surface of substrate through the silica template (Fig. 2(a)). Before surfactant removal, a redox signal of ferricyanide anion is not observed in its original redox potential range before surfactant $(\mathrm{CTAB})$ removal but the current appears after subsequent surfactant removal. This indicates that ferricyanide ions are accessible to the ITO surface only after surfactant removal. The decreased redox current compared to result on bare ITO electrode implies restricted mass transport of ferricyanide anions by silica template. The restriction is ascribed to the repulsive electrostatic 

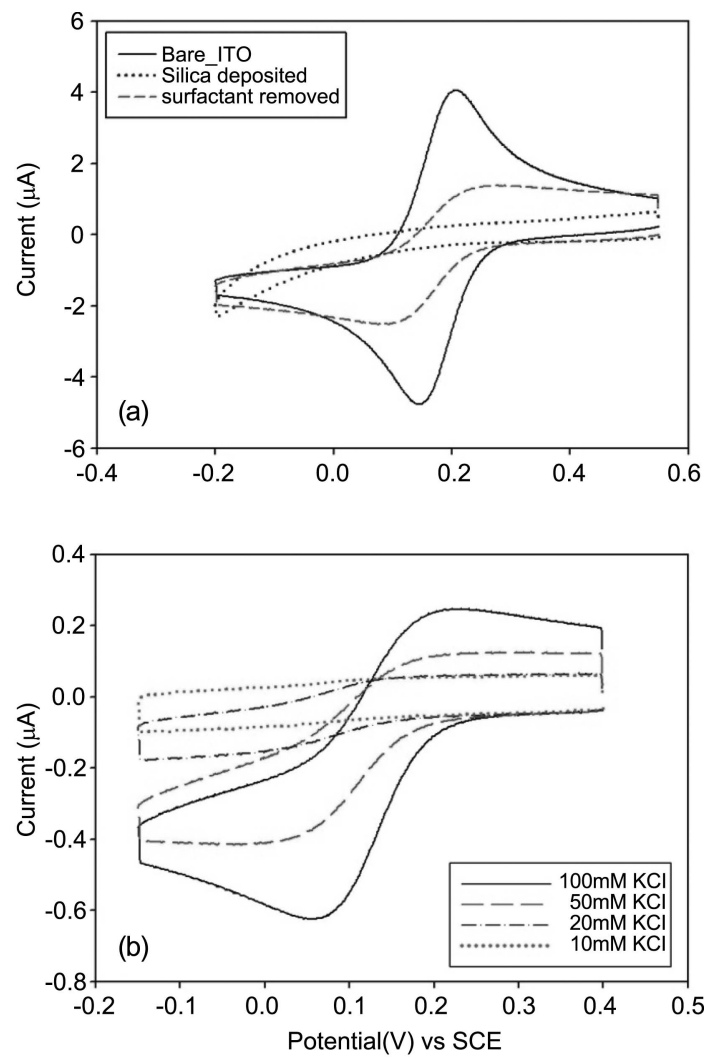

Fig. 2. Cyclic voltammogram of $\mathrm{Fe}(\mathrm{CN})_{6}{ }^{3-/ 4-}$ redox couple (a) in $0.1 \mathrm{M} \mathrm{KCl}$ solution on silica/ITO composite electrode before/after surfactant removal and on bare ITO electrode. (b) 0.01-0.1 M KCl solution on silica-ITO composite electrode. Scan rate : $50 \mathrm{mV} / \mathrm{s}$. Surface area of electrode is $0.28 \mathrm{~cm}^{2}$.

interactions between negatively-charge silica pore walls and ferricyanide anions. ${ }^{4,5)}$ The redox current becomes smaller with decreasing supporting electrolyte concentration (Fig. 2(b)), which reflects the increased unfavorable interaction at lower ionic strength. In brief, all these characteristics unequivocally prove the formation of the vertically oriented silica template. ${ }^{4,5)}$

\subsection{Deposition of Ppy into silica template}

Ppy deposition on silica-ITO composite electrode from pyrrole monomer solution is demonstrated by $\mathrm{CV}$ and compared with the result on bare ITO electrode (Fig. 3(a)). The onset potential of Ppy deposition on silica template is slightly decreased compared with bare ITO, while overall current is much smaller.

Fig. 3(b) shows the voltage profile observed in galva-

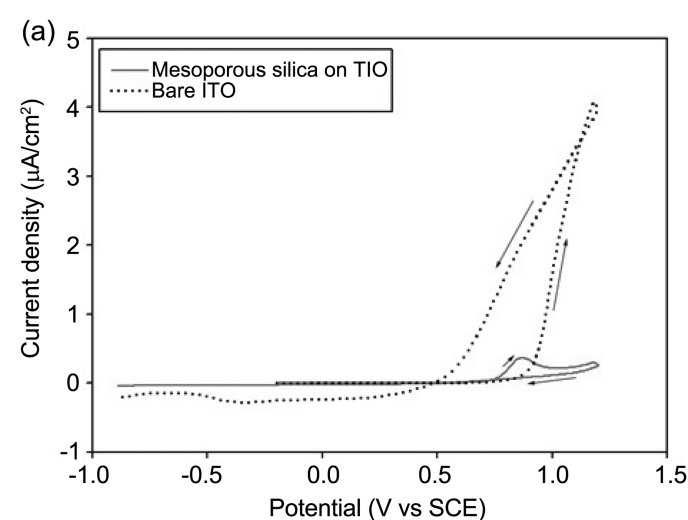

(b)

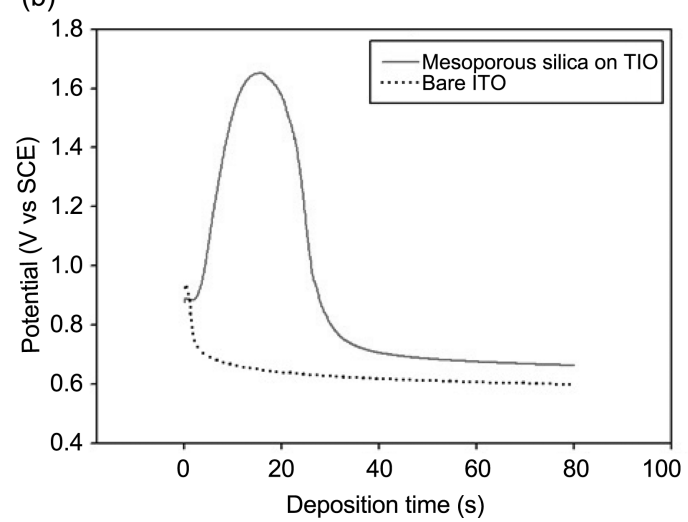

Fig. 3. (a) Cyclic voltammogram at scan rate $0.1 \mathrm{~V} / \mathrm{s}$ and (b) Potential-time curve on galvanostatic deposition at the current density, $57 \mathrm{~A} / \mathrm{cm}^{2}$ on silica/ITO composite electrode. $0.1 \mathrm{M}$ pyrrole, $0.2 \mathrm{M} \mathrm{KCl}$ solution was used.

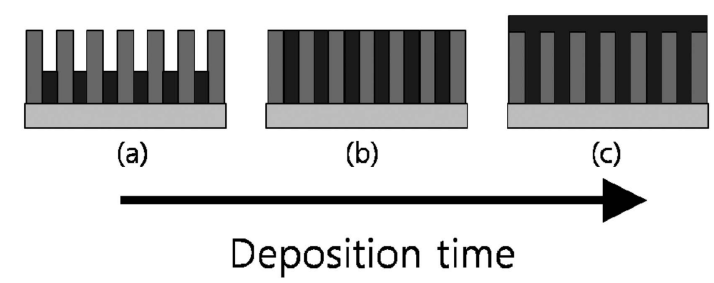

Fig. 4. Hypothesis of progress of Ppy growth.

nostatic Ppy deposition. Ppy polymerization is initiated at lower potential on the silica template compared to bare ITO electrode. The potential required to maintain constant current increases as the deposition progress. After passing through the maximum, the voltage decreases to flatten around $0.7 \mathrm{~V}$. The voltage profile in Fig. 3(b) can be related with the degree of deposition (Fig. 4). It seems that higher potential is required to grow Ppy nanowires through the mesopores, because mass transfer for Ppy 
growth is restricted compared with bare ITO (Fig. 4(a)). After the pores are filled with Ppy nanowires completely (Fig. 4(b)), further Ppy growth takes place at larger area, requiring less overpotential (Fig. 4(c)) than before. Similar features are also observed in growth of templated gold nanowires. $^{11)}$

3.3. Redox behavior of Ppy nanowires embedded in silica template

Redox behavior of Ppy nanowires in vertically aligned silica template on ITO electrode is assessed by CV. The amount of Ppy deposited is controlled by the total charge consumed for the electropolymerization. ${ }^{12)}$ Ppy has been galvanostatically deposited until the potential-time curve passes the maximum. About $2.15 \mathrm{mC}$ was consumed to deposition process. The same amount of charge was used on bare ITO to prepare a counterpart. Fig. 5(a) is cyclic voltammogram of Ppy nanowires embedded in silica/ITO electrode and compared with the silica/ITO electrode without Ppy. Reversible reduction and oxidation current observed in the potential range of $-0.2 \mathrm{~V} \sim 0 \mathrm{~V}$. It is interesting to note that Ppy nanowires exhibits redox activity as embedded in silica template on electrode without template removal. In the case of Ppy deposited on bare ITO (Fig. 5(b)), the redox peaks are not clearly

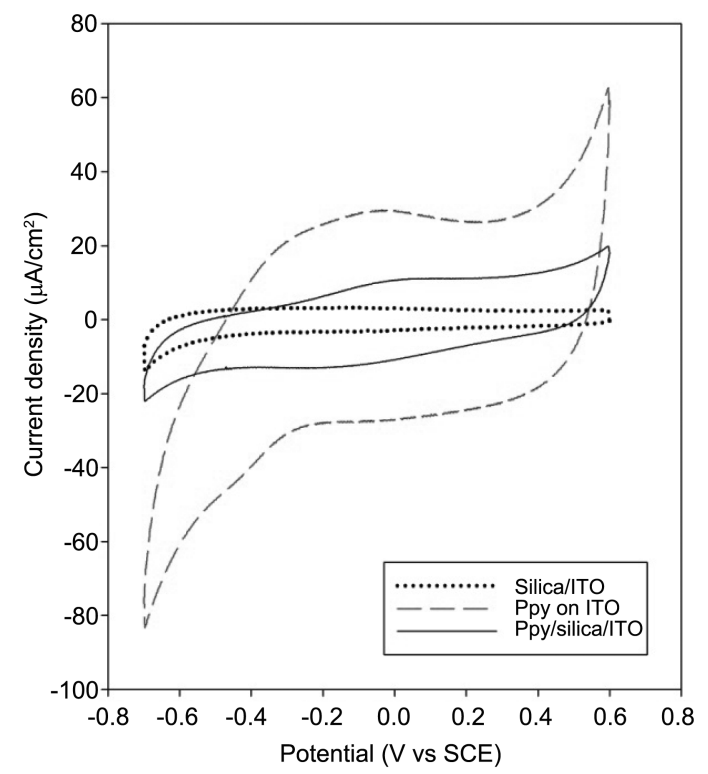

Fig. 5. Cyclic voltammogram of Ppy/silica/ITO composite electrode and Ppy/ITO in 0.2 M KCl solution. Scan rate : $0.1 \mathrm{~V} / \mathrm{s}$. observed, due to the increased non-faradaic charging current. The redox behavior of Ppy nanowires after removal of silica template (i.e., by HF etching) and the origin of the different redox behavior deserve further study.

\section{Conclusion}

In this study, Ppy nanowires embedded in vertically aligned mesoporous silica template have been prepared by electrochemical method on ITO electrode. The composite electrode was demonstrated by scanning electron microscope and electrochemical experiment. Ppy nanowires in the composite electrode were found to exhibit reversible electroactivity.

\section{Acknowledgment}

This work was supported by the Nano/Bio Science \& Technology Program (2010-0008213) of the Ministry of Education, Science and Technology, and the Basic Science Research Program through a National Research Foundation of Korea (NRF) grant funded by the Ministry of Education, Science and Technology (2010-0001951). This paper was supported by Research Fund, Kumoh National Institute of Technology for H. Lee.

\section{References}

1. R. Liu and S. B. Lee, " $\mathrm{MnO}_{2} / \mathrm{Poly}$ (3,4-ethylenedioxythiophene) Coaxial Nanowires by One-Step Coelectrodeposition for Electrochemical Energy Storage", J. Am. Chem. Soc., 130, 2942 (2008).

2. F. Cheng, M. Zhang, and H. Wang, "Fabrication of Polypyrrole Nanowire and Nanotube Arrays", Sensors, 5 , 245 (2005).

3. A. Walcarius, E. Sibottier, M. Etienne, and J. Ghanbaja, "Electrochemically assisted self-assembly of mesoporous silica thin films", Nat. Mater, 6, 602 (2007).

4. A. Goux, M. Etienne, E. Aubert, C. Lecomte, J. Ghanbaja, and A. Walcarius, "Oriented Mesoporous Silica Films Obtained by Electro-Assisted Self-Assembly (EASA)", Chem. Mater., 21, 731 (2009).

5. A. Goux, J. Ghanbaja, and A. Walcarius, "Prussian Blue electrodeposition within an oriented mesoporous silica film: preliminary observations" J. Mater. Sci., 44, 6601 (2009).

6. Y. Li, B. Wang, H. Chen, and W. Feng, "Improvement of the electrochemical properties via poly(3,4-ethylenedioxythiophene) oriented micro/nanorods" J. Power Sources, 195, 3025 (2010).

7. C. J. Brinker, Y. Lu, A.Sellinger, and H. Fan, "Evaporation- 
Induced Self-Assembly: Nanostructures Made Easy“, $A d v$. Mater, 11, 579 (1999).

8. P. J. Vruinsma, A. Y. Kim, J. Liu, and S. Baskaran, "Mesoporous Silica Synthesized by Solvent Evaporation: Spun Fibers and Spray-Dried Hollow Spheres", Chem. Mat., 9, 2507 (1997).

9. N. Raman, M. Anderson, and C. Brinker, "TemplateBased Approaches to the Preparation of Amorphous, Nanoporous Silicas" Chem. Mater, 8, 1682 (1996).

10. C. Brinker, C. J. Dunphy, et al, "Morphological control of surfactant-templated metal oxide films", Curr. Opin. Colloid Interface Sci., 11, 126 (2006).

11. W. Kautek, S. Reetz, and S. Pentzen, "Template electrodeposition of nanowire arrays on gold foils fabricated by pulsed-laser deposition", Electrochim. Acta., 40, 1461 (1995).

12. S. Basak, K. Rajeshwar, and M. Kaneko, "Ion binding by poly \{pyrrole-co-[3-(pyrrol-1-yl)propanesulfonate]\} thin films", Anal. Chem., 62, 1407 (1990). 